\title{
The applicability of spike time dependent plasticity to development
}

\author{
Daniel A. Butts ${ }^{1 *}$ and Patrick O. Kanold ${ }^{1,2 *}$ \\ Department of Biology and Program in Neuroscience and Cognitive Science, University of Maryland, College Park, MD, USA \\ Institute for Systems Research, University of Maryland, College Park, MD, USA
}

Edited by:

Per Jesper Sjöström, University College London, UK

Reviewed by:

Marla B. Feller, University of California USA

Carlos D. Aizenman, Brown University, USA

David Stellwagen, McGill University,

Canada

\section{${ }^{*}$ Correspondence:}

Daniel A. Butts and Patrick O. Kanold, Department of Biology, University of Maryland, 1118 Biosciences Research Building, College Park, MD 20742, USA. e-mail:dab@umd.edu and pkanold@ umd.edu
Spike time dependent plasticity (STDP) has been observed in both developing and adult animals. Theoretical studies suggest that it implicitly leads to both competition and homeostasis in addition to correlation-based plasticity, making it a good candidate to explain developmental refinement and plasticity in a number of systems. However, it has only been observed to play a clear role in development in a small number of cases. Because the fast time scales necessary to elicit STDP, it would likely be inefficient in governing synaptic modifications in the absence of fast correlations in neural activity. In contrast, later stages of development often depend on sensory inputs that can drive activity on much faster time scales, suggesting a role in STDP in many sensory systems after opening of the eyes and ear canals. Correlations on fast time scales can be also be present earlier in developing microcircuits, such as those produced by specific transient "teacher" circuits in the cerebral cortex. By reviewing examples of each case, we suggest that STDP is not a universal rule, but rather might be masked or phased in, depending on the information available to instruct refinement in different developing circuits. Thus, this review describes selected cases where STDP has been studied in developmental contexts, and uses these examples to suggest a more general framework for understanding where it could be playing a role in development.

Keywords: cortex, thalamus, timescale, burst, subplate, teacher, microcircuit

\section{INTRODUCTION}

Spike time dependent plasticity (STDP) was initially reported in both developing and adult animals (Bell et al., 1997; Magee and Johnston, 1997; Markram et al., 1997; Zhang et al., 1998; Feldman, 2000). As a result, STDP has always been thought to have a role in development: reflecting a more general conception that rules of synaptic plasticity, or "learning rules", may be conserved across developing and adult brains, as well as across different brain areas.

The prospect of STDP functioning during development is captivating for experimentalists and theorists alike, because it offers opportunities to connect in vivo neural activity to observable system-level organization of neural connections. Making this connection in a developing system is facilitated by the presence of observable organizing principles in these systems that describe the establishment of connections, such as the refinement of retinal ganglion cells (RGC) axons into eye specific layers/regions in the lateral geniculate nucleus (LGN) and superior colliculus (SC) as well as formation and plasticity of ocular dominance columns in the visual cortex (Katz and Shatz, 1996; Crair, 1999; Sur and Rubenstein, 2005). In contrast, the patterns of connectivity established during adult functions such as learning and memory do not have easily observable system-level organizational patterns associated with them, and as a result, in these contexts it is much more difficult to gauge the effects of STDP (or other learning rules).

A second reason the prospect of STDP acting during development is so compelling is the nature of STDP itself, compared with other types of synaptic plasticity. Realistic spike trains are generally composed of complex temporal patterns, and these "natural" patterns of activity do not fit into the categories of tetanic stimulation, theta burst, pairing, and other simplified experimental contexts that synaptic learning rules are usually studied with. The basis of STDP on the timing of single spikes potentially provides a formula for taking an arbitrary set of pre- and postsynaptic activity and predicting what the effect of such activity patterns on the synaptic efficacy will be. Furthermore, because of its close apposition of windows for potentiation and depression, as described below, several theoretical studies have shown that STDP implicitly introduces both competition and homeostatic regulation between the inputs to a given neuron (Song et al., 2000; Tegner and Kepecs, 2002; Izhikevich and Desai, 2003; Watt and Desai, 2010), potentially explaining what have been traditionally thought of as separate aspects of developmental plasticity.

Thus, STDP studies during development present the possibility of understanding the role of synaptic plasticity in vivo, whereby known activity patterns might be combined with known changes in connectivity and arrive at a known outcome. However, though appealing, STDP may be only one form of synaptic plasticity that governs development. As we describe in this review, STDP fits more broadly into the category of Hebbian plasticity (Caporale and Dan, 2008), but with the additional requirement for relationships of neural activity between presynaptic afferents and postsynaptic targets on the order of $10 \mathrm{~ms}$. As a result, for STDP to yield consistent results at a given synapse, neuronal activity must contain information at these "fast" time scales. Likewise, the tight temporal correlation requirement limits the applicability of STDP in cases where there is no such tight pairing between pre- and postsynaptic activity. As a result, below, we describe 
where STDP likely does not work (the developing retinogeniculate synapse), where it likely does (the developing somatosensory cortical microcircuit), and where it could (early visual cortical development). By bringing together these disparate studies, both experimental and theoretical, in multiple brain areas, we hope to focus investigations from a simple study of mechanisms independent of what might be accessed in an animals' development, to mechanisms that are likely to play a role in particular developmental contexts.

\section{"HEBBIAN" DEVELOPMENT AND STDP}

Based on developmental studies in which either sensory experience or neuronal activity was modulated, activity-dependent development is generally thought to be governed by Hebbian plasticity: "cells that fire together wire together" (Katz and Shatz, 1996). This principle was originally postulated to explain learning in the guise of classical conditioning (Hebb, 1949), and reflects the idea that the brain is attempting to internalize the causal nature of the world in connections between individual neurons. Such a principle also offers a compelling explanation for many aspects of observed development. In particular, during stages of development thought to be driven by activity, many axonal arbors - in particular those that project from one brain area or cortical layer to another (and thus are observably distinct) - become refined from initially more diffuse projections (Figure 1A). Given that these diffuse projections are coarsely organized (most likely by molecular cues; Sur and Rubenstein, 2005), any local presynaptic activity patterns will lead to the largest correlations in activity between a given presynaptic neuron and postsynaptic neurons at the center of its axonal arbor. As a result, Hebbian principles predict the strengthening of the appropriate connections in such contexts, and thus have been postulated to govern developmental refinement throughout.

Because of its requirement for correlation between pre- and postsynaptic neurons, STDP is considered a "Hebbian" learning rule as well (Caporale and Dan, 2008). However, its sensitivity to fast time scales and strict temporal order between inputs and targets also seems to be at odds with looser causality described above. Namely, if presynaptic afferents onto the postsynaptic neuron are weak and diffuse - as is often the case earlier in development there will only be very subtle correlations over the short time scales required for STDP-based potentiation. Even in the case where preand postsynaptic firing rates are correlated, there will still be many spike pairs falling into the window for depression (Figure 1B). As a result, depending on the relative strength of induced potentiation versus depression, this could be very inefficient at strengthening the correct synapses, or even counterproductive (Lu et al., 2006).

However, while STDP is clearly inefficient in this context, its sensitivity to strict temporal order leads to effects that are consistent with other aspects of developmental refinement. In particular, simple Hebbian learning rules are not sufficient to explain developmental refinement alone, without further rules governing synaptic strength. For example, the overall amount of synaptic input to a given neuron is thought to be regulated, such that a given neuron maintains a relatively constant amount of activity over time. Homeostatic regulation is presumably present at all times but is likely to be particularly important during development, when synaptic remodeling typically does not preserve the total number of inputs (Chen and Regehr, 2000; Turrigiano and Nelson, 2000, 2004; Chandrasekaran et al., 2007; Goel and Lee, 2007). Synaptic competition, whereby the strengthening of one input is balanced by the weakening of other inputs is also postulated to be important for refinement and plasticity during development. For example, closure of one eye during the critical period of visual development will often increase the number of inputs into the cortex representing the open eye, at the expense of the inputs representing the closed eye (Wiesel and Hubel, 1963).

In a series of theoretical studies, STDP has been shown to implicitly result in synaptic competition and homeostatic regulation of postsynaptic firing (Song et al., 2000), and thus could explicitly
A

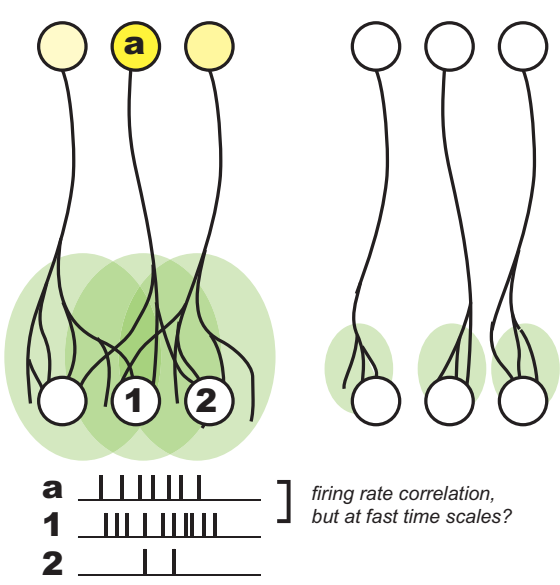

FIGURE 1 | Development of coarse patterning of CNS connections. (A) Projections between different brain regions often are initially larger and diffuse (left), but refine over time in an activity dependent manner (right). Because of initial organization of this map, local activity patterns generally drive the highest postsynaptic firing in the center of a given afferent (a) projection (compare activity of neuron \#1 with \#2). As a result, Hebbian principles generally can explain this

B
Weak connection,
many neurons correlated
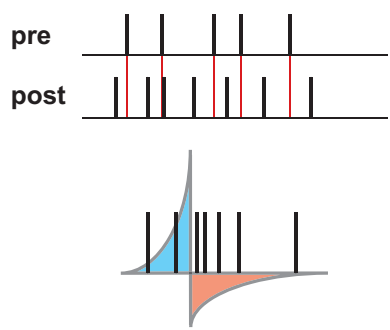

During coarse organization, earlier development?
Strong connection, few neurons correlated

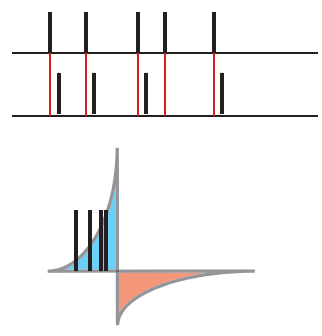

During refinement of more specific connectivity? refinement. (B). However, without a strong connection between pre- and postsynaptic neurons, STDP might be quite inefficient at supporting such Hebbian development, such as earlier in development when connections are weak and diffuse (left). When connections become stronger, the relationship between preand postsynaptic activity might develop much more precise correlations, when presynaptic spikes have a larger role in driving postsynaptic activity (right). 
explain synaptic refinement in a handful of developmental contexts (Song and Abbott, 2001). These elements arise because the balance of potentiation and depression is skewed in favor of depression for uncorrelated inputs. What this means is that as one input gets stronger, it will have an increased probability of driving a postsynaptic spike. Because these postsynaptic spikes follow the presynaptic spike that preceded it - as well as those correlated with this input - it will further strengthen these correlated inputs. At the same time, inputs that are uncorrelated will on average be depressed, and thus potentiation of correlated inputs implicitly occurs at the expense of uncorrelated inputs: resulting in competition. Furthermore, this competition for the timing of the postsynaptic spike will also result in a form of homeostatic regulation of postsynaptic activity. For example, if the amount of input activity to a postsynaptic neuron increases, there will initially be more postsynaptic spikes, leading to more weakening overall given the overall predominance of depression over potentiation postulated above. This implicitly acts to down-regulate the total amount of input into the neuron. Likewise, when there are fewer postsynaptic spikes, there will be less depression overall, making it easier for a given input to become potentiated through weaker correlations with postsynaptic activity. This implicit homeostasis of STDP is likely only one aspect of overall homeostatic regulation of neurons, as discussed in more detail in an accompanying review in this issue (Watt and Desai, 2010).

Thus, from a theoretical perspective, STDP is an excellent candidate to explain many elements of synaptic plasticity during development. Yet, at the same time, in many developmental systems, STDP is not appropriately tuned to the time scales of their observed neural activity, and thus would be inefficient in many contexts, if not completely ineffective. With this background, we will thus discuss several examples of observed developmental plasticity, and its relationship to STDP.

\section{A LIMITED ROLE FOR STDP AT THE DEVELOPING RETINOGENICULATE SYNAPSE}

The developing retinogeniculate system provides an example where the neural activity itself is not structured to take advantage of the temporal sensitivity of STDP, and thus serves as a counter-example to the universal applicability of STDP. Before eye opening, spontaneous correlated activity sweeps across the developing retina, called "retinal waves" (Meister et al., 1991; Feller et al., 1996). Such retinal wave activity has been implicated in coarse system-level organizational refinement in the visual pathway, including eyesegregation and retinotopic refinement in the LGN (Katz and Shatz, 1996; Penn et al., 1998; Grubb et al., 2003; Pfeiffenberger et al., 2006), SC (Simon et al., 1992; Shah and Crair, 2008), and retinotopic refinement in the visual cortex (Cang et al., 2005).

Changes in efficacy of retinogeniculate synapses can be induced by tetanic stimulation (Mooney et al., 1993; Ziburkus et al., 2009), showing that this synapse can be plastic, albeit in the context of an unphysiological stimulation paradigm (Figure 2A, top). However, these studies did not make clear how plasticity would evince in the context of the well-studied activity patterns present in the RG system during this period, and it was tempting to imagine that development at the retinogeniculate synapse might also be governed by STDP, as has been observed at the corresponding retinotectal synapse of Xenopus (Zhang et al., 1998). However, it was unclear if such a learning rule could take advantage of the activity present in the retina at this time: retinal waves themselves consist of RGC bursts over a second or more, travel over small domains of the retina over the course of several seconds (Feller et al., 1997), and result in bursts in the LGN that also can last for a second or more (Mooney et al., 1996). Because of the slow speed of wave propagation, the information content of this activity has very little information available at fast $(<20 \mathrm{~ms})$ time scales, with the bulk present at time scales of $500 \mathrm{~ms}$ or more (Butts and Rokhsar, 2001). Thus, both the activity and coarse refinement thought to be driven by this activity reflect the situation described in Figure 1, where STDP based on millisecond time scales would be very inefficient at driving developmental refinement.

As a result, synaptic plasticity was measured using physiological 1-s bursts of with different latencies (Figure 2B), revealing a broad temporal window for potentiation surrounded by weak depression windows (Figure 2C). This defines burst-time dependent plasticity (BTDP; Butts et al., 2007a), in which the amount of synaptic plasticity depends on the latency differences between pre- and postsynaptic bursts. Subsequently, BTDP has also been observed in the developing SC (Shah and Crair, 2008). The induction protocol of BTDP offered the ability to test for an underlying spike-based learning rule using activity patterns like those seen in vivo. A collection of spike-time latencies could be measured for each pair of pre- and postsynaptic bursts, and correlating the observed plasticity induced by the burst pairing with the histogram of spike latencies led to two main conclusions about a spike-based learning rule. First, as predicted above, the observed amounts of potentiation can only be explained if the depressive window of STDP is neglected. Second, the burst-rule, with a time scale of $1 \mathrm{~s}$, can actually be predicted by a spike-coincidence rule on time scales $\sim 50 \mathrm{~ms}$ (Figure 2D), provided there is no sensitivity to temporal order.

It is important to note that while such results might seem to suggest that STDP does not explain the observed plasticity at the retinogeniculate synapse, they are in fact consistent with the detailed "interaction rules" for STDP discovered using more complicated spike trains in other preparations (Sjostrom et al., 2001; Froemke et al., 2006). In cases where multiple spike pairs are involved, the resulting plasticity is consistent with ignoring the spike pairs that predict depression, in favor of those predicting potentiation. In the context of more complex spike trains, this implies that the strict order of spike pairs might be less important, and that STDP could resemble a more conventional Hebbian rule.

In this way, one might imagine that differences in neural activity driving different developmental programs might select for different types of Hebbian plasticity. Though it is not known whether STDP functions at the LGN synapse in isolated-spike contexts, the existence of spike-interaction rules that make the synaptic plasticity more appropriate for the longer time scales of spike trains with multiple spikes raises the possibility that STDP might be part of a broader continuum of Hebbian plasticity that acts during development, and its function either actively adjusts through, for example, neuromodulation (Seol et al., 2007), or passively adjusts through explicit interaction rules (Sjostrom et al., 2001; Froemke et al., 2006) to efficiently drive developmental plasticity in each context. 
A

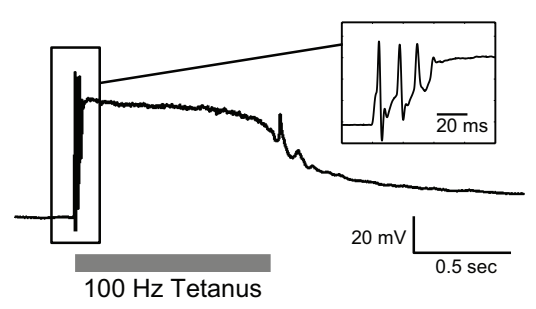

B

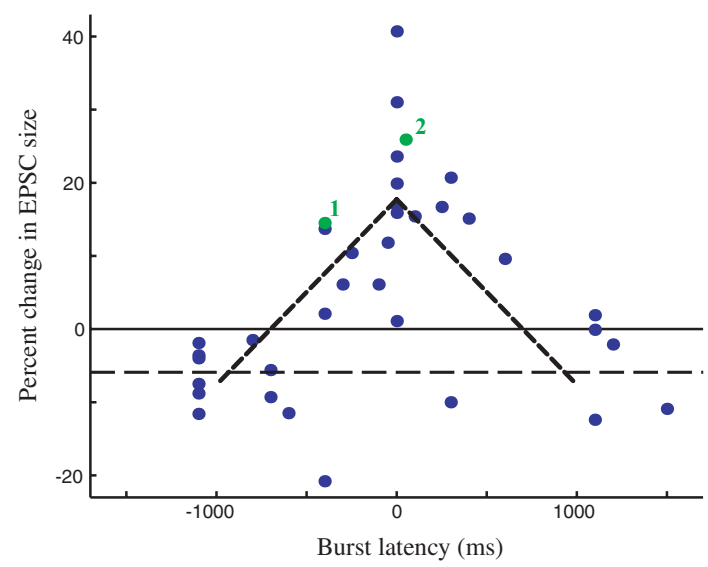

FIGURE 2 | Burst time dependent - but not spike time dependent learning rule at the developing retinogeniculate synapse. (A) The effect of tetanic stimulation on the voltage of postsynaptic LGN neuron, resulting in an unphysiological state, especially in comparison to experiments that approximate in activity in vivo (C). (B) The burst time dependent learning rule, demonstrating a tent-shape peaked at 0 latency, and decreasing to slight depression after $1 \mathrm{~s}$ on either side. Each dot shows an individual measurement in the percent change in peak postsynaptic current before and
C
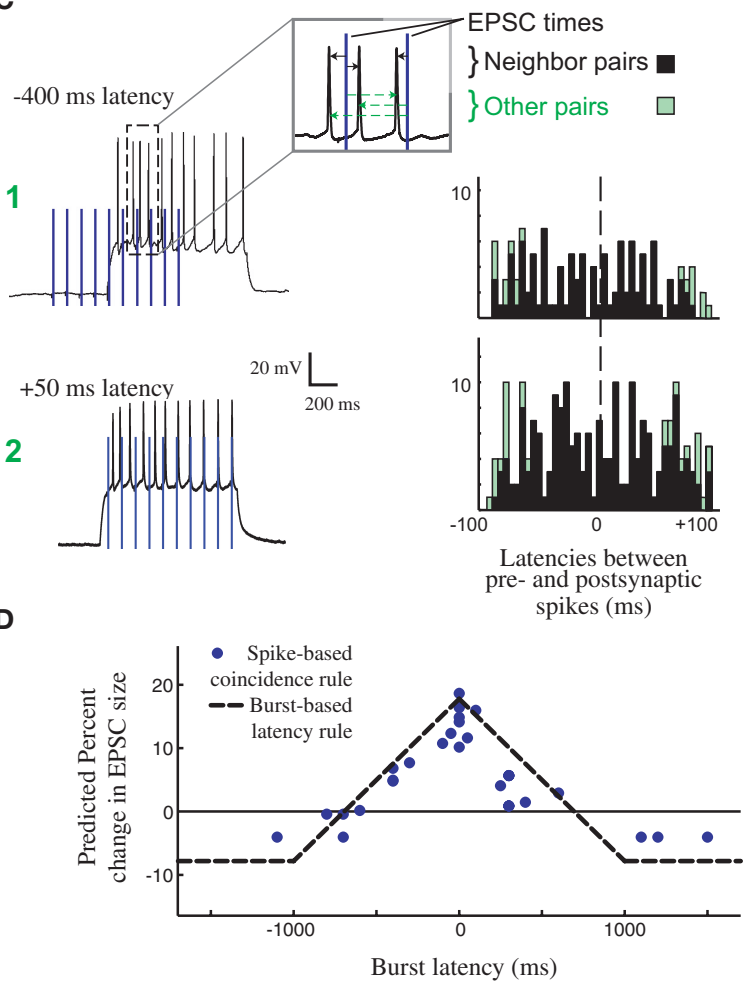

after burst stimulation. (C) The timing of all pairs of pre- and postsynaptic spikes during the burst-based protocol can be tabulated (right) in order to test whether the observed effects can be attributed to some form of STDP. Two example bursts with two different latencies are shown (left), leading to the observed plasticity reported in (B, \#1, and \#2). (D) The predicted learning rule using a single-spike based coincidence detector, which explains the burst-based rule much better than STDP. All panels are reproduced from Butts et al. (2007a).

\section{A ROLE FOR STDP IN CORTICAL MICROCIRCUIT PLASTICITY}

The inefficiency of "isolated-spike" STDP in the canonical example of development depicted in Figure 1 does not necessarily apply to other aspects of activity dependent development. In other contexts, correlations between neural activity between afferents and postsynaptic targets can be quite tight - especially when the activity is driven by sensory stimulation, or more generally in the case of microcircuits driven by common input. In these situations, the Hebbian principles thought to govern coarse development at coarse time scales might likewise be applied at finer scales through STDP, and a more conventional Hebbian learning rule such as BTDP described would not be able to take advantage of information at finer time scales that distinguishes one input from another.

One of the most established examples of STDP playing a clear role in development is sensory driven map plasticity at excitatory layer 4 (L4) to layer 2/3 (L2/3) synapses in the barrel cortex during the critical period (Foeller and Feldman, 2004). Over this period of development, deprivation of sensory stimulation to a set of whiskers induces rapid map plasticity such that L2/3 neurons in the corresponding barrels begin responding to adjacent non-deprived barrels, resulting from weakening of the deprived barrel inputs and strengthening of non-deprived barrel inputs (Foeller and Feldman, 2004).
A model for how STDP leads to barrel map plasticity in $\mathrm{L} 2 / 3$ relies on the relative latencies between direct feed-forward input from L4 and horizontal connections between L2/3 neurons in different barrels (Figure 3). Initially, L2/3 neurons are driven by L4 neurons of the same barrel (left), but after deprivation, the L2/3 neurons in the deprived barrel can still be driven through horizontal connections from other barrels, coordinated by sensory activity that can correlate inputs of multiple barrels. The ability of horizontal connections to drive L2/3 activity directly will lead to short latencies between these horizontal connections consistent with STDP-mediated strengthening. In the meantime, the direct input from L4 is only spontaneously active, which cannot reliably drive postsynaptic responses. Because synaptic input that is uncorrelated with postsynaptic firing leads to net depression (Feldman, 2000) - presumably because of a larger window for depression than strengthening - the direct L4 to L2/3 connection in the deprived barrel will weaken.

This model of STDP-mediated plasticity is validated by two further observations. First, in vivo studies demonstrate that neural activity in the barrels is largely composed of few spikes (Higley and Contreras, 2006; Vijayan et al., 2010), and the relative latencies between the involved neurons in behaviorally relevant conditions 
A

\section{deprived non-deprived}

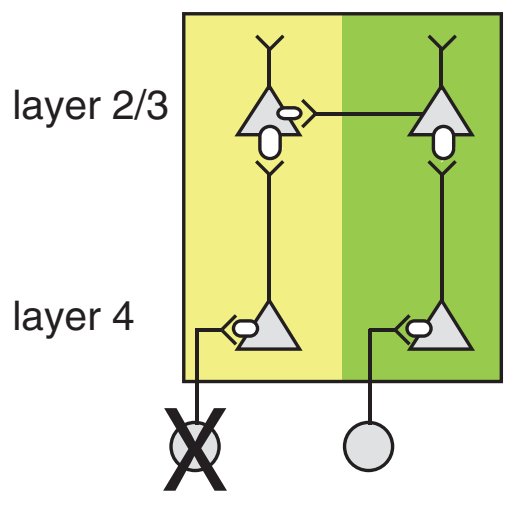

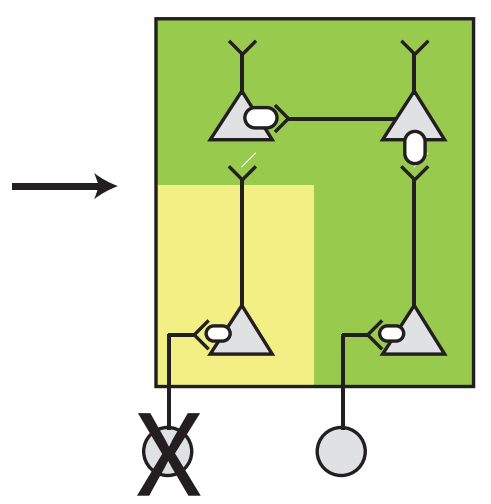

B

correlated layer 4 spikes with deprived layer $2 / 3$

non-deprived

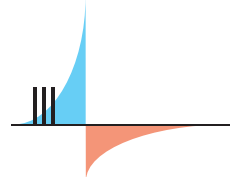

deprived

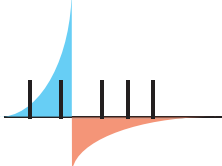

FIGURE 3 | Barrel cortex plasticity. (A) Local circuit changes in barrel cortex following whisker deprivation. Shown are layer 4 (L4) and layer 2/3 (L2/3) cells and their excitatory synaptic connections. The size of the receptors indicates the strength of the synapse. Yellow shading indicates cells driven by the deprived barrel and green shading indicates cells driven by the non-deprived barrel.

Reducing sensory input to one barrel by whisker deprivation (deprived) (left) results in weakening of $L 4$ to $L 2 / 3$ synapses and strengthening of horizontal connections in $L 2 / 3$ originating from a non-deprived barrel (right) such that $L 2 / 3$ in the deprived region of barrel cortex will respond strongly to the stimulation of a non-deprived whisker. (B) L2/3 neurons in deprived barrel cortex show correlated activity with $L 4$ neurons in the non-deprived region of barrel cortex leading to strengthening of horizontal connections. Correlations with the deprived $L 4$ are weak as the deprived whisker provides little sensory input. The potentiating and weakening windows of STDP rule are indicated in blue and red shading. is on the fast time scales of STDP (Armstrong-James et al., 1992; Allen et al., 2003). Second, a causal relationship between deprivation in vivo and effects on observed STDP in the relevant barrel in vitro has been observed (Feldman, 2000; Allen et al., 2003; Foeller and Feldman, 2004; Jacob et al., 2007), suggesting a direct role of STDP in barrel map plasticity.

\section{STDP AND THE FRAMEWORK OF "TEACHER CIRCUITS"}

The circuits involved in barrel cortex plasticity described above fit into a general framework called a teacher circuit (Song and Abbott, 2001; Friedel and van Hemmen, 2008), where a weak "nascent" input that it is not able to drive the postsynaptic "student" neuron alone, is paired with a stronger "teacher" input or inputs (Figure 4, left). Analogous to the classical conditioning paradigm of Hebbian plasticity, if the teacher input is paired within a certain time window with the nascent input and can drive precisely timed postsynaptic spikes, the spikes will fall into the potentiating window of STDP and the nascent inputs become strengthened, i.e., the student learns the input. Once the nascent input is able to drive postsynaptic spikes on its own, it will induce further selfpotentiation. Additionally, if it more efficiently drives postsynaptic activity, it will occur ahead of the teacher input, placing teacher spikes in the depression window of STDP and result in weakening of the teacher input. As a result, the teacher will withdraw as the student learns other inputs, with the more efficient connections taking over.

The process is reversed in the context of deprivation, such as whisker removal (described above). Under deprivation conditions - because the learned input no longer drives postsynaptic spikes - the teacher again begins to dominate the firing of the student and re-potentiate. As a result, spontaneous firing from the learned inputs will now more often fall into larger depression windows and weaken (Figure 4B).
A key requirement for this teacher circuit to work is that the timing of teacher-evoked postsynaptic spikes must be closely correlated with the inputs to be learned, such that their spikes fall within the potentiating window of the STDP rule. In the scenario of the barrel cortex described above, the timing relationships are due to coactivation of neighboring barrels while whisking. Because the teacher pathway involves an additional synapse that sensory input must traverse, teacher-evoked spikes in the student neurons will always occur after direct L4 inputs. Such a scenario might also be present in the case of monocular deprivation (MD) in the visual cortex (Song and Abbott, 2001), and may underlie observation of initial remapping in $\mathrm{L} 2 / 3$ followed by $\mathrm{L} 4$ in visual deprivation experiments at later ages (Trachtenberg et al., 2000).

Thus, in this microcircuit, precisely timed sensory-evoked activity can travel through two different pathways, and STDP serves to establish connectivity that most efficiently represents the causation. In this instance, horizontal connections are able to take over in the case of deprivation, and allow the cortex to process sensory stimuli even without direct input. As described below, when deprivation ends, the same STDP rules will reestablish the previous connectivity, flexibly supporting cortical processing of sensory stimuli.

\section{HYPOTHESIZED ROLE OF STDP IN VISUAL CORTICAL DEVELOPMENT}

For reasons explored above, STDP has not been observed or probed in most developmental systems. However, the framework above sets a foundation for understanding more generally where STDP might play a part in guiding development. For example, similar circuit topologies are also present in early development. The formation of feed-forward connections between the thalamus and the cortex is thought to be mediated by a distinct class of cells: subplate neurons, which form one of the first functional cortical circuits (Kanold, 
A

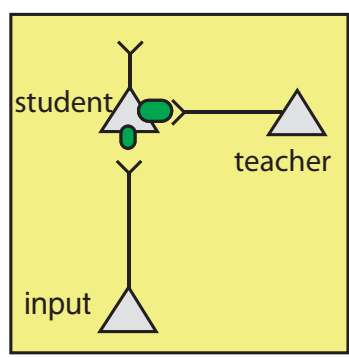

initial development

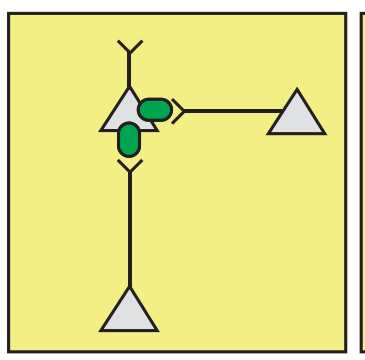

B

deprivation

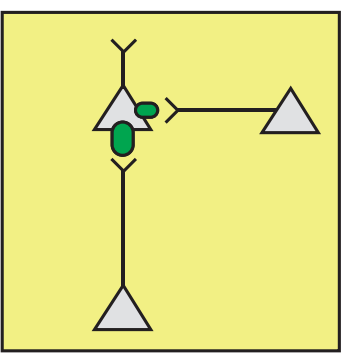

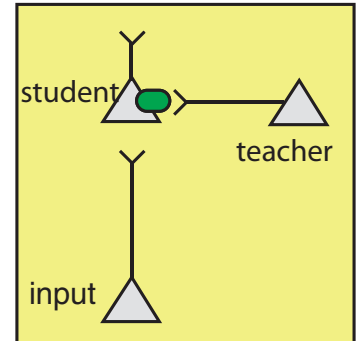

\section{spike correlations of student neuron with input and teacher neuron}

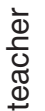
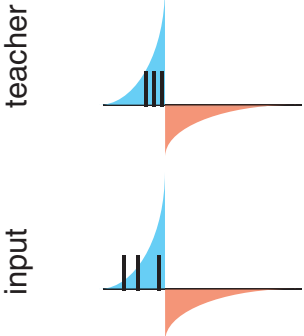

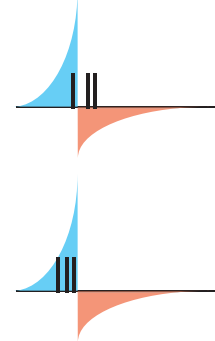

FIGURE 4 |The framework of the "teacher circuit." (A) The teacher circuit consists of a student neuron with two inputs: the teacher (inside box) and nascent inputs to be learned (inside box). Left. Initially, the teacher has a strong connection (top), and its activity is paired with a weaker input, leading to spikes from both inputs falling in the potentiation window of STDP (bottom). The potentiating and weakening windows of STDP rule are indicated in blue and red shading. Middle: The pairing of the teacher and nascent input strengthens the
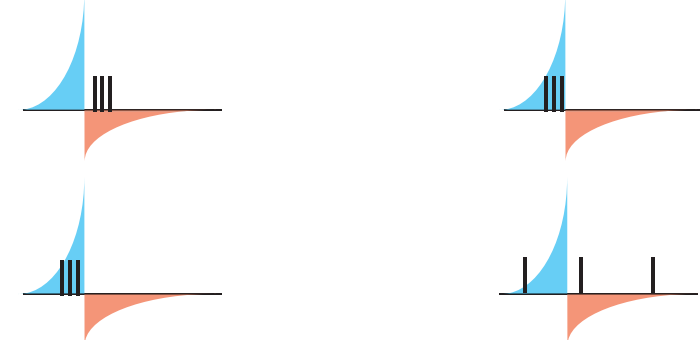

2009; Zhao et al., 2009). Subplate neurons excite L4 neurons and themselves receive inputs from thalamic axons before these axons reach L4 (Shatz and Luskin, 1986; Kanold, 2009; Zhao et al., 2009) (Figure 5A). Thus subplate neurons provide a strong feed-forward projection of thalamic inputs to L4 before thalamic inputs to L4 strengthen. Furthermore, removal of subplate neurons prevents the maturation of connections between the LGN and visual cortex, and the emergence of ocular dominance columns in visual cortex (Ghosh and Shatz, 1992; Kanold et al., 2003; Kanold and Shatz, 2006). However, why the cortex would need a transiently expressed circuit to guide thalamocortical connectivity, and how the specific connectivity and activity of subplate neurons plays a role in this development, is currently not well understood (Kanold and Luhmann, 2010).

The structure of the circuit formed by the subplate and thalamocortical afferents, and L4 neurons is not unlike the teacher circuit described above. Subplate neurons at early ages have a high input resistance, allowing them to spike with minimal latency (Zhao et al., 2009). In addition to the rapid firing onset, subplate neurons at young ages fire only few spikes to sustained inputs, thus marking the onset of a stimulus. Thus, subplate neurons potentially can convert neuronal bursts that are prevalent in the early stages of the visual pathway due to retinal waves (Meister et al., 1991; Feller et al., 1996) into sparse spike trains allowing the analysis with STDP. nascent input (top), but also results in some teacher spikes in the LTD window (bottom), resulting in eventual weakening of the teacher. Right. With the nascent input "learned", teacher spikes fall completely in the weakening window (bottom), reinforcing a weak teacher input and strong learned input. (B) During deprivation, the learned input can no longer drive postsynaptic firing, and learned input becomes uncorrelated with postsynaptic spikes (bottom), and the teacher begins to drive student firing again and strengthens.
Furthermore, subplate activity is likely able to drive spiking activity in L4, because L4 neurons at relevant ages also have high input resistances (Zhao et al., 2009).

As a result, it has been suggested that STDP might govern circuit maturation in an analogous way to the teacher circuit described above (Kanold and Shatz, 2006). In this case subplate neurons play the role of the teacher, with a strong input onto L4 neurons (the students), and the nascent inputs are the thalamocortical afferents (Figure 5A). Because subplate neurons are driven by thalamic activity, subplate mediated depolarization of L4 cells occurs after direct thalamocortical input to $\mathrm{L} 4$, meaning that presynaptic thalamic input will fall into the potentiation window of STDP (Figure 5A). In the meantime, the strong subplate input is slightly delayed due to the disynaptic pathway from the thalamus. Thus, the initially strong subplate inputs will lead to the strengthening of thalamic inputs, which would replace the subplate as the main driving input to the cortex through the framework discussed above.

This framework can also explain the effects of the subplate ablation (Ghosh and Shatz, 1992; Kanold et al., 2003; Kanold and Shatz, 2006) (Figure 5B). Without the subplate present, thalamic activity would no longer drive L4 neurons with the appropriate time scales to fall into the potentiation window of STDP, analogously to the deprived barrel inputs described above. If activity levels in both eyes are equal, inputs representing both eyes are weakened equally over 
A

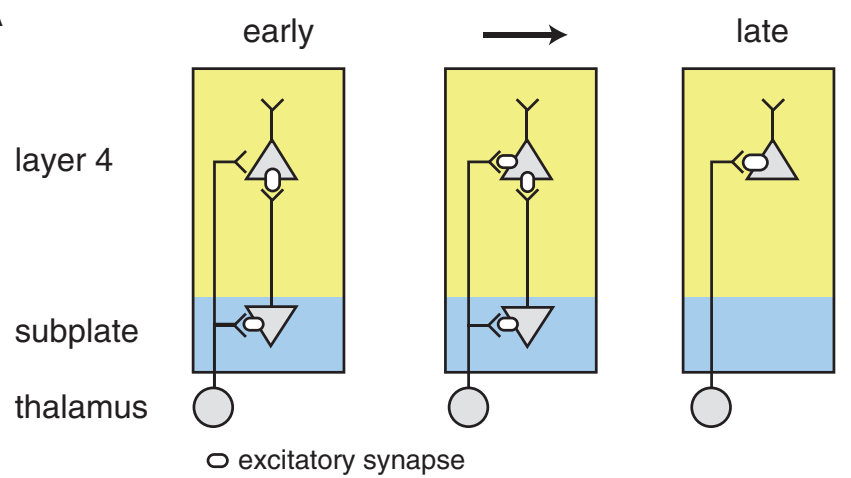

B

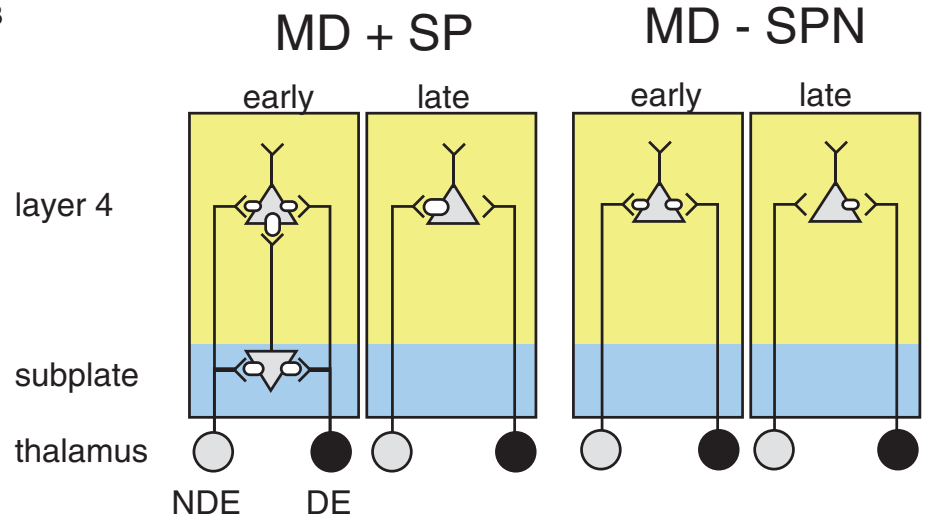

correlated spikes with layer 4
early $\quad$ late
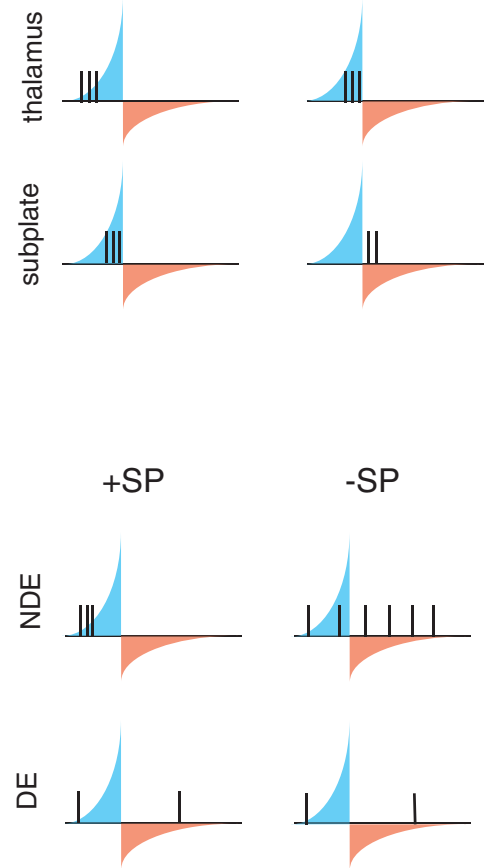

FIGURE 5 |The influence of subplate on thalamocortical microcircuit development and plasticity. Diagramed are subplate neurons and the connections of thalamic neurons with subplate neurons and layer 4 neurons. The strength of the excitatory synaptic connections is indicated by the size of the receptor. (A) Changing subplate circuits (left) from early to late in development when subplate neurons die. Blue shading indicates the subplate while yellow indicates the cortical plate. Spike correlations between layer 4 and its inputs from subplate and thalamus at these developmental stages are plotted at right. The STDP rule is superimposed illustrating the changing correlations over development. The potentiating and weakening windows of STDP rule are indicated in blue and red shading. (B) Effects of subplate neurons on the outcome of monocular deprivation (MD) on ocular dominance. Left panels show strengthening of the non-deprived eye (NDE) inputs and weakening of deprived eye (DE) inputs when subplate is present. Right panels show weakening of the NDE inputs and retention of DE inputs when subplate is absent, leading to paradoxical ocular dominance plasticity. Spike correlation at right show that layer 4 activity is uncorrelated with thalamic activity when subplate is removed, leading to selective weakening of NDE. time and no refinement of ocular dominance would be observed (Kanold and Shatz, 2006) matching observations from physiological experiments (Kanold et al., 2003). In contrast, if activity levels in both eyes are unequal, for example during MD thalamic axons representing the open eye would have a larger amount of uncorrelated activity with $\mathrm{L} 4$ neurons than axons representing the closed eye (Kanold and Shatz, 2006) (Figure 5B). Thus, over time the projections representing the open eye would disappear while projections representing the closed eye would be retained, paralleling experimental observations (Kanold and Shatz, 2006).

One key property of the subplate circuit discussed above is its transient existence, which is consistent with it no longer being useful once the students have "learned" the correct thalamocortical inputs. One other transient aspect of the developing cortical circuit is the existence of depolarizing GABAergic circuits (Ben-Ari, 2002). Such circuits may also act as "teachers" during early development, and just as the subplate disappearance removes feed-forward excitatory drive - the switch of GABA signaling from depolarizing to hyperpolarizing might occur after the relevant developmental phase.
In conclusion, STDP provides an elegant explanation of multiple aspects of the development of the cortical microcircuit, driven by a combination of spontaneous and sensory-evoked activity though the stages of cortical development. Because of the feed-forward nature of the involved microcircuit, relevant information about the desired presynaptic targets are contained in the fast time scales of STDP, and the difference between potentiation and depression is determined by the difference between monosynaptic versus disynaptic connections. In such a situation, it is clear how STDP could play a direct role in development.

\section{RELEVANCE OF STDP IN DIFFERENT STAGES OF DEVELOPMENT}

The primary difference between retinal-wave-driven development of the retinogeniculate system and microcircuit development in the cortex is the time scale of the information contained in activity in these different systems. Information that can instruct the coarse system-level organization of retinogeniculate development is contained in the seconds-long time scales of retinal wave propa- 
gation (Butts and Rokhsar, 2001). In contrast, the development of cortical microcircuitry may predominantly rely on differences in timing between latencies of monosynaptic versus disynaptic connections. Though arising through different mechanisms, such fast time scales are also typical of visually driven activity of thalamocortical afferents, due to the precise response properties of LGN neurons during natural visual stimulation (Butts et al., 2007b).

As a result, STDP may also be relevant when activity in the visual system is visually evoked, rather than spontaneously generated. In fact, one of the first places that STDP was observed was the developing retinotectal system of Xenopus (Zhang et al., 1998), which is visually responsive throughout development. As reviewed in a companion article (Richards et al., 2010), such a rule can be exploited to train retinotectal cells to become motion selective using visual input (Engert et al., 2002), drive receptive field refinement through pair of visual activity and stimulation (Vislay-Meltzer et al., 2006), or alter the temporal responses of tectal cells by recruiting recurrent circuits (Pratt et al., 2008). Similar studies have demonstrated STDP-like effects in later stages of development and adult. For example, temporally pairing related auditory or visual stimuli with each other over fast time scales can elicit in changes in the stimulus tuning of cortical neurons, and these changes depended on temporal order and follow the general temporal STDP shape (Yao and Dan, 2001; Dahmen et al., 2008). Further evidence for STDP in driving synaptic remodeling in adult comes from studies of shifts in receptive fields following retinal lesions (Young et al., 2007). In these cases the activity present in the system contains information at fast time scales, and the observed shifts are consistent with the strict temporal ordering of the STDP rule.

Thus, over the course of development, the formation of the functional circuitry involves formation, refinement, and plasticity of connections on vastly different spatial scales, from the development of topographic projections between areas (e.g., between retina and LGN) to development of specific microcircuits between nearby neurons, for example in the cerebral cortex. The development of

\section{REFERENCES}

Allen, C. B., Celikel, T., and Feldman, D. E. (2003).Long-term depression induced by sensory deprivation during cortical map plasticity in vivo. Nat. Neurosci. 6, 291-299.

Armstrong-James, M., Fox, K., and DasGupta, A. (1992). Flow of excitation within rat barrel cortex on striking a single vibrissa. J. Neurophysiol. 68, 1345-1358.

Bell, C. C., Han, V. Z., Sugawara, Y., and Grant, K. (1997). Synaptic plasticity in a cerebellum-like structure depends on temporal order. Nature 387, 278-281.

Ben-Ari, Y. (2002). Excitatory actions of gaba during development: the nature of the nurture. Nat. Rev. Neurosci. 3 , 728-739.

Butts, D. A., Kanold, P. O., and Shatz, C. J. (2007a). A burst-based "Hebbian" learning rule at retinogeniculate synapses links retinal waves to activity-dependent refinement. PLoS Biol. 5, e61. doi:10.1371/journal. pbio.0050061.

Butts, D. A., Weng, C., Jin, J., Yeh, C. I., Lesica, N.A., Alonso, J.M., and Stanley, G. B. (2007b). Temporal precision in the neural code and the timescales of natural vision. Nature 449, 92-95.

Butts, D. A., and Rokhsar, D. S. (2001). The information content of spontaneous retinal waves. J. Neurosci. 21, 961-973.

Cang, J., Renteria, R. C., Kaneko, M., Liu, X., Copenhagen, D. R., and Stryker, M. P. (2005). Development of precise maps in visual cortex requires patterned spontaneous activity in the retina. Neuron 48, 797-809.

Caporale, N., and Dan, Y. (2008). Spike timing-dependent plasticity: a Hebbian learning rule. Annu. Rev. Neurosci. 31, 25-46. connections on different spatial scales occurs at different times and involves different activity patterns. While spontaneous activity patterns drive early coarse refinement, sensory-evoked activity drives later fine scale development.

Given that STDP extracts information at fast time scales, the canonical form of STDP, with close apposition of windows for potentiation and depression, might operate at stages in development that are dominated by sensory inputs after opening of the eyes and ear canals. At earlier stages of development when activity is still spontaneously generated, development that relies on STDP (such as cortical microcircuit development) might use teacher circuits such as those formed by subplate neurons, which can transform long lasting spontaneous waves in precisely timed spike signals. Or, as suggested above in the case of the retinogeniculate system, the same underlying mechanisms that result in STDP might be active in a different form, more appropriate for the time scales relevant for instructing development.

\section{CONCLUSION}

Spike time dependent plasticity likely supports the development and plasticity of several neuronal circuits, explaining a variety of elements that must be present for refinement of neuronal connectivity. For a full understanding of circuit plasticity one needs to take into account both the synaptic learning rule, the activity patters present, and the timescales of their information content. As a result, STDP is likely to play a role in the development of circuits where information is present at fast timescales $(<20 \mathrm{~ms})$ or where specific teacher circuits aid the development of fast temporal correlation, as is done by the feed-forward connections formed by subplate neurons. However, STDP is unlikely to play a role in development in systems where high spike rates are present or where information is only present at longer time scales, such as the developing retinogeniculate system.

\section{ACKNOWLEDGMENT}

Patrick O. Kanold is supported by NIH R01DC009607.
Chandrasekaran, A. R., Shah, R. D., and Crair, M. C. (2007). Developmental homeostasis of mouse retinocollicular synapses. J. Neurosci. 27, 1746-1755.

Chen, C., and Regehr, W. G. (2000) Developmental remodeling of the retinogeniculate synapse. Neuron 28 , 955-966.

Crair, M. C. (1999). Neuronal activity during development: permissive or instructive? Curr. Opin. Neurobiol. 9, 88-93.

Dahmen, J. C., Hartley, D. E., and King, A. J. (2008). Stimulus-timingdependent plasticity of cortical frequency representation. J. Neurosci. 28, 13629-13639.

Engert, F., Tao, H.W.,Zhang, L. I., and Poo, M. M. (2002). Moving visual stimuli rapidly induce direction sensitivity of developing tectal neurons. Nature 419, 470-475.
Feldman, D. E. (2000). Timing-based LTP and LTD at vertical inputs to layer II/ III pyramidal cells in rat barrel cortex. Neuron 27, 45-56.

Feller, M. B., Butts, D. A., Aaron, H. L., Rokhsar, D. S., and Shatz, C. J. (1997). Dynamic processes shape spatiotemporal properties of retinal waves. Neuron 19, 293-306.

Feller, M. B., Wellis, D. P., Stellwagen, D., Werblin, F. S., and Shatz, C. J. (1996). Requirement for cholinergic synaptic transmission in the propagation of spontaneous retinal waves. Science 272, 1182-1187.

Foeller, E., and Feldman, D. E. (2004). Synaptic basis for developmental plasticity in somatosensory cortex. Curr. Opin. Neurobiol. 14, 89-95.

Friedel, P., and van Hemmen, J. L. (2008). Inhibition, not excitation, is the key to multimodal sensory integration. Biol. Cybern. 98, 597-618. 
Froemke, R. C., Tsay, I. A., Raad, M., Long, J.D., and Dan, Y. (2006). Contribution of individual spikes in burst-induced long-term synaptic modification. $J$. Neurophysiol. 95, 1620-1629.

Ghosh, A., and Shatz, C. J. (1992). Involvement of subplate neurons in the formation of ocular dominance columns. Science 255, 1441-1443.

Goel, A., and Lee, H. K. (2007). Persistence of experience-induced homeostatic synaptic plasticity through adulthood in superficial layers of mouse visual cortex. J. Neurosci. 27, 6692-6700.

Grubb, M. S., Rossi, F. M., Changeux, J. P., and Thompson, I. D. (2003). Abnormal functional organization in the dorsal lateral geniculate nucleus of mice lacking the beta 2 subunit of the nicotinic acetylcholine receptor. Neuron 40, 1161-1172.

Hebb, D. (1949). The Organization of Behavior. New York: Wiley.

Higley, M. J., and Contreras, D. (2006). Balanced excitation and inhibition determine spike timing during frequency adaptation. J. Neurosci. 26, 448-457.

Izhikevich, E. M., and Desai, N. S. (2003). Relating STDP to BCM. Neural. Comput. 15, 1511-1523.

Jacob, V., Brasier, D. J., Erchova, I., Feldman, D., and Shulz, D. E. (2007). Spike timing-dependent synaptic depression in the in vivo barrel cortex of the rat. J. Neurosci. 27, 1271-1284.

Kanold, P. O. (2009). Subplate neurons: crucial regulators of cortical development and plasticity. Front. Neuroanat. 3:16. doi:10.3389/neuro.05.016.2009.

Kanold, P. O., Kara, P., Reid, R. C., and Shatz, C. J. (2003). Role of subplate neurons in functional maturation of visual cortical columns. Science 301, 521-525.

Kanold, P. O., and Luhmann, H. J. (2010). The subplate and early cortical circuits. Annu. Rev. Neurosci. 33, 23-48.

Kanold, P. O., and Shatz, C. J. (2006). Subplate neurons regulate maturation of cortical inhibition and outcome of ocular dominance plasticity. Neuron 51, 627-638.

Katz, L. C., and Shatz, C. J. (1996). Synaptic activity and the construction of cortical circuits. Science 274, 1133-1138.

Lu, H. C., Butts, D. A., Kaeser, P. S., She, W. C., Janz, R., and Crair, M. C. (2006)
Role of efficient neurotransmitter release in barrel map development. $J$. Neurosci. 26, 2692-2703.

Magee, J. C., and Johnston, D. (1997). A synaptically controlled, associative signal for Hebbian plasticity in hippocampal neurons. Science 275, 209-213.

Markram, H., Lubke, J., Frotscher, M. and Sakmann, B. (1997). Regulation of synaptic efficacy by coincidence of postsynaptic APs and EPSPs. Science $275,213-215$

Meister,M., Wong, R. O., Baylor, D. A., and Shatz, C. J. (1991). Synchronous bursts of action potentials in ganglion cells of the developing mammalian retina. Science 252, 939-943.

Mooney, R., Madison, D. V., and Shatz, C. J. (1993). Enhancement of transmission at the developing retinogeniculate synapse. Neuron 10, 815-825.

Mooney, R., Penn, A. A., Gallego, R., and Shatz, C. J. (1996). Thalamic relay of spontaneous retinal activity prior to vision. Neuron 17, 863-874.

Penn, A. A., Riquelme, P. A., Feller, M. B., and Shatz, C. J. (1998). Competition in retinogeniculate patterning driven by spontaneous activity. Science 279 , 2108-2112.

Pfeiffenberger, C., Yamada, J., and Feldheim, D.A. (2006). Ephrin-As and patterned retinal activity act together in the development of topographic maps in the primary visual system. $J$. Neurosci. 26, 12873-12884.

Pratt, K. G., Dong, W., and Aizenman, C. D. (2008). Development and spike timing-dependent plasticity of recurrent excitation in the Xenopus optic tectum. Nat. Neurosci. 11,467-475.

Richards, B. A., Aizenman, C. D., and Akerman, C. J. (2010). In vivo spiketiming-dependent plasticity in the optic tectum of Xenopus laevis. Front. Syn. Neurosci. 2:7. doi:10.3389/ fnsyn.2010.00007.

Seol, G. H., Ziburkus, J., Huang, S., Song, L., Kim, I. T., Takamiya, K., Huganir, R. L., Lee, H. K., and Kirkwood, A. (2007). Neuromodulators control the polarity of spike-timing-dependent synaptic plasticity. Neuron 55, 919-929.

Shah, R. D., and Crair, M. C. (2008). Retinocollicular synapse maturation and plasticity are regulated by correlated retinal waves. J. Neurosci. 28 , 292-303.
Shatz, C. J., and Luskin, M. B. (1986). The relationship between the geniculocortical afferents and their cortical target cells during development of the cat's primary visual cortex. J. Neurosci. 6 3655-3668.

Simon, D. K., Prusky, G. T., O’Leary, D. D., and Constantine-Paton, M. (1992). $\mathrm{N}$-methyl-D-aspartate receptor antagonists disrupt the formation of a mammalian neural map. Proc. Natl. Acad. Sci. U.S.A. 89, 10593-10597.

Sjostrom, P. J., Turrigiano, G. G., and Nelson, S. B. (2001). Rate, timing, and cooperativity jointly determine cortical synaptic plasticity. Neuron $32,1149-1164$.

Song, S., and Abbott, L. F. (2001). Cortical development and remapping through spike timing-dependent plasticity. Neuron 32, 339-350.

Song, S., Miller, K. D., and Abbott, L. F. (2000). Competitive Hebbian learning through spike-timing-dependent synaptic plasticity. Nat. Neurosci. 3 919-926.

Sur, M., and Rubenstein, J. L. (2005). Patterning and plasticity of the cerebral cortex. Science 310, 805-810.

Tegner, J., and Kepecs, A. (2002). Sliding threshold correlational learning rule follows from spike-timing dependent plasticity. In Society for Neuroscience Annual Meeting, 32, 152.115 (Abstract).

Trachtenberg, J. T., Trepel, C., and Stryker, M. P. (2000). Rapid extragranular plasticity in the absence of thalamocortical plasticity in the developing primary visual cortex. Science 287 2029-2032.

Turrigiano, G. G., and Nelson, S. B. (2000) Hebb and homeostasis in neuronal plasticity. Curr. Opin. Neurobiol. 10, 358-364.

Turrigiano, G. G., and Nelson, S. B. (2004) Homeostatic plasticity in the developing nervous system. Nat. Rev. Neurosci. 5, 97-107.

Vijayan, S., Hale, G. J., Moore, C. I. Brown, E. N., and Wilson, M. A. (2010). Activity in the barrel cortex during active behavior and sleep. $J$ Neurophysiol. 103, 2074-2084.

Vislay-Meltzer, R. L., Kampff, A. R., and Engert, F. (2006). Spatiotemporal specificity of neuronal activity directs the modification of receptive fields in the developing retinotectal system. Neuron 50, 101-114.

Watt, J., and Desai, N. (2010). Homeostatic plasticity and STDP: keeping a neuron's cool in a fluctuating world. Front. Syn. Neurosci. 2, 1-16. doi:10.3389/ fnsyn.2010.00005

Wiesel, T. N., and Hubel, D. H. (1963). Single-cell responses in striate cortex of kittens deprived of vision in one eye. J. Neurophysiol. 26, 1003-1017.

Yao, H., and Dan, Y. (2001). Stimulus timing-dependent plasticity in cortical processing of orientation. Neuron 32, 315-323.

Young, J. M., Waleszczyk, W. J., Wang, C., Calford, M. B., Dreher, B., and Obermayer, K. (2007). Cortical reorganization consistent with spike timing-but not correlation-dependent plasticity. Nat. Neurosci. 10, 887-895.

Zhang, L. I., Tao, H. W., Holt, C. E., Harris, W. A., and Poo, M. (1998). A critical window for cooperation and competition among developing retinotectal synapses. Nature 395, 37-44.

Zhao, C., Kao, J. P., and Kanold, P. O. (2009). Functional excitatory microcircuits in neonatal cortex connect thalamus and layer 4. J. Neurosci. 29, 15479-15488.

Ziburkus, J., Dilger, E. K., Lo, F. S., and Guido, W. (2009). LTD and LTP at the developing retinogeniculate synapse.J. Neurophysiol. 102, 3082-3090.

Conflict of Interest Statement: The authors declare that the research was conducted in the absence of any commercial or financial relationships that could be construed as a potential conflict of interest.

Received: 22 February 2010; paper pending published: 10 March 2010; accepted: 27 June 2010; published online: 19 July 2010. Citation: Butts DA and Kanold PO (2010) The applicability of spike time dependent plasticity to development. Front. Syn. Neurosci. 2:30. doi: 10.3389/ fnsyn.2010.00030

Copyright (C) 2010 Butts and Kanold. This is an open-access article subject to an exclusive license agreement between the authors and the Frontiers Research Foundation, which permits unrestricted use, distribution, and reproduction in any medium, provided the original authors and source are credited. 